\title{
Abiotic stress responsive cis-regulatory elements (CREs) in rice (Oryza sativa L.) and other plants
}

\author{
Sangram Keshari Lenka ${ }^{1,2}$ and Kailasah C. Bansal ${ }^{1}$ \\ ${ }^{1}$ National Research Centre on Plant Biotechnology, Indian Agricultural Research Institute, \\ New Delhi, 110012 \\ ${ }^{2}$ Current Address: TERI-Deakin NanoBiotechnology Centre, The Energy and Resources \\ Institute, New Delhi, 110003
}

Keywords: ABRE, cis-elements, rice

\begin{abstract}
Capability of crop plants to adjust to the adverse environmental conditions in a spatiotemporal fashion is critical for their survival and maintaining agricultural productivity. Genetic engineering efforts for improving tolerance to diverse abiotic stresses in crop plants using well characterised stress-inducible promoter elements have proven to be advantageous. Combinatorial interactions of cis-acting DNA elements in the promoters with trans-acting protein factors are key processes governing spatio-temporal gene expression. It is becoming increasingly evident that targeted modification of molecular genetic network is feasible, for exploiting the potential of specific abiotic stress responsive element and its corresponding master regulatory genes via plant genetic engineering. The importance of inducible promoters in agricultural crop improvement is enormous; hence it is very crucial to characterise inducible promoters from plant genome sequence data bases on a large scale. We will briefly discuss here abiotic stress responsive cis-acting elements and their role in abiotic stress regulated gene expression.
\end{abstract}

\section{Introduction}

Due to their sessile life style, plants are only able to survive by their ability to build up fast and highly adaptive responses to diverse environmental stresses. These adaptive mechanisms involve a complex network for perception and transmission of stress signals to initiate cellular responses by changes in enzyme activity, gene expression, and transport of molecules across membranes (Boyer, 1982; Mittler, 2006). Abiotic stress tolerance in plants involves diverse genetic mechanisms and versatile molecular adaptive processes (Bohnert et al., 1995). Therefore, capability of crop plants to adjust to the adverse environmental conditions in a 
spatio-temporal fashion is critical for their survival and maintaining agricultural productivity. The greatest challenge for humanity in the next few decades will be to increase and sustain agricultural productivity without degrading the ecosystem. Deep understanding about abiotic stress responses in crop plants is vital for the continuous development of rational precision breeding programs as well as for devising transgenic strategies for developing improved stress tolerant crops plants. During the last two decades, tremendous advancement in recombinant DNA technology has enabled us to introduce traits of agronomic importance into the crop plants. However, it is still difficult to introduce a complex trait such as drought tolerance regulated by multiple genes. Some unique regulatory genes (transcription factor encoding genes) controlling several downstream cellular responses have been successfully integrated into some of the plant species to improve tolerance to environmental stresses including drought.

Plant genetic engineering efforts for improving tolerance to diverse abiotic stresses in crop plants using well characterised stress-inducible promoter elements and master regulatory genes (transcription factors) have proven to be advantageous (Bhatnagar-Mathur et al., 2008; Yamaguchi-Shinozaki and Shinozaki, 2005). We mainly discuss here functional characterization of ABA responsive cis-acting elements (ABREs) in plant species.

\section{Cis-elements: Driving Gene Expression}

Cis-regulatory elements basically include core promoter elements, proximal promoter elements, and long range regulatory elements. RNA polymerase II, its other subunits and different trans-acting protein factors (transcription factors) recognise the core promoter elements and other functional DNA sequences to initiate transcription in response to internal and external cues. TATA box, the initiator element and other downstream promoter elements are well characterised components of the core promoter element. A promoter becomes nonfunctional if the orientation and the distance between the core elements and the transcription start sites altered. However, the enhancer elements, classified under long range regulatory elements will still (but not always) have the ability to activate gene expression. Apart from these cis-elements, the insulators function as long-range regulatory elements and may function as boundary mark between heterochromatin and euchromatin or may function for blocking enhancer activity to regulate the accurate expression of the adjacent genes (BurgessBeusse et al., 2002).

Based on the nature of promoters, they are basically divided into three types: 
i.) Constitutive promoters ii.) Tissue-specific promoters iii.) Inducible promoters Among these three groups of promoters, inducible promoters up-regulates the corresponding gene expression in response to internal and external signals. The interest of researchers in inducible system of promoters is increasing for two reasons: (i) to understand precise functional role of genes under stress and (ii) to allow crop plants to have "auto-regulatory" expression of trans-genes under versatile environmental conditions (Reynolds, 1999). However, in some instances, the ectopic expression (leaky expression) and pleiotropic expression of genes negatively affect the use of inducible promoters (Aoyama, 1999). The importance of inducible promoters in agricultural crop improvement is enormous; hence it is very crucial to characterise inducible promoters from plant genome sequence data bases on a large scale. Complete understanding of the functional elements within the inducible promoters responsible for driving gene expression is very crucial to engineer synthetic promoters of interest to drive expression of the potential transgenes (Venter, 2007).

\section{Abiotic Stress Responsive Transcriptional Gene Regulation}

Transcription factor is a protein that binds to specific cis-regulatory element (CRE) and thereby regulates transcription of genetic information from DNA to primary RNA transcript (Latchman, 1997). Molecular genetic analysis and genetic engineering for abiotic stress tolerance during the past one-decade revealed that transcriptome engineering (use of master switch genes such as signaling proteins and transcription factors) is a viable option for enhancing abiotic stress tolerance of crop plants. Due to the complex and versatile nature of abiotic stress response of plants, multiple stress receptors rather than a single receptor, are more likely to be responsible for perception of the stress. One of the important lessons learnt is that the regulons and sub-regulons of master regulators vary from species to species and thus determine the differential sensitivity of these species to abiotic stresses. Hence, masterregulatory genes from closely related species appear to be better candidate genes rather than that from distant species.

Plants respond to environmental cues at molecular level by altering expression of different sets of genes (Qureshi et al., 2007; Tran et al., 2007). Expressions of such genes are mainly regulated through transcriptional control process, while post-transcriptional and posttranslational processes also play a crucial role. Transcriptional control machinery appears to be conserved among plant species (Hakimi et al., 2000; Hirt et al., 1990). It is well established from different experiments over past decades that promoters containing a particular cis-element respond to a specific trigger (Viswanathan and Zhu, 2002) 
(Chinnusamy et al., 2003; Viswanathan and Zhu, 2002; Yamaguchi-Shinozaki and Shinozaki, 2005; Zhou et al., 2007). Combinatorial interactions of cis-acting DNA elements in the promoters with trans-acting protein factors are key processes governing spatio-temporal gene expression (Bustos et al., 1991; Hartmann et al., 2005; Hauffe et al., 1993). At an organism level, vast array of molecular genetic networks are operational in a very complex and dynamic mode. Complete understanding of the molecular genetic networks is a long cherished goal of system biologists (Chinnusamy et al., 2004; Li et al., 2006). Till today, several groups of abiotic stress inducible transcription factor families have been characterised from crop plants; these primarily include AP2/ERF, bZIP, MYB and MYC, Zinc-finger proteins, HD-ZIP, NAC, etc. In order to dissect out regulatory activity of promoters in response to abiotic stresses, deep understanding of various transcription factors and their subregulons are indispensible.

Occurrence of specific abiotic stress responsive cis-regulatory element is the hallmark of abiotic stress responsive promoter element. It is becoming increasingly evident that targeted modification of molecular genetic network is feasible, for exploiting the potential of specific abiotic stress responsive element and its corresponding master regulatory genes via plant genetic engineering.

\section{Abiotic Stress Responsive cis-Acting Elements}

Promoters regulate gene expression by specific cis-regulatory elements either by enhancing (enhancers) or repressing (repressors) the transcription efficiency in response to environmental stresses. Several abiotic stress responsive cis-acting elements are well characterised in different plant species, such as ABA responsive element (ABRE) in response to $\mathrm{ABA}$ and drought responsive element/C-repeat sequence (DRE/CRT) receptive to high salinity, dehydration and low temperature (Yamaguchi-Shinozaki and Shinozaki, 2005).

\section{ABA Responsive cis-Acting Elements (ABRE)}

The ABRE cis-element was originally discovered in EmBP-1b promoter of wheat (Triticum aestivum) as 8-base pair (bp) sequence (CACGTGGC) (Guiltinan et al., 1990). Subsequently, several stress-inducible promoters have been characterised having ABRE with a conserved core element -ACGT. Molecular dissection experiments like deletion and linker scanning analysis have pinpointed the ABA responsive elements (ABREs), also termed as G-box, Cbox or G-box/C-box hybrids within promoters of the ABA responsive genes. The ACGT core nucleotide sequence within ABRE, acts as a binding site for bZIP family transcription factors 
governing transcriptional regulation of ABA responsive genes (Guiltinan et al., 1990; Hattori et al., 2002; Mundy et al., 1990; Ross and Shen, 2006; Shen and Ho, 1995). ABREs are also coupled to the non-ACGT coupling elements like CE1, CE3, DRE, O2S, motif III or ACGT core containing ABRE itself (Hobo et al., 1999; Shen et al., 1996; Singh, 1998). Table-1 summarises the list of ACGT and non-ACGT core containing ABA responsive elements validated by various wet-lab experiments. We identified and validated the rice specific ABRE (CGMCACGTGB) using integrated partial interactive methods and expectation maximization algorithm (MEME) as our top prediction for ABA responsive element (Lenka et al., 2009). Non ACGT core containing ABREs have also been reported (Abe et al., 1997), and named as MYBR and MYCR. These cis-elements act like weak enhancer elements responsible for ABA responsive induction in promoters of $R D 22$ genes. Hence, all these $\mathrm{ABA}$ responsive components listed in Table-1 are significant hardware of ABA-dependent pathway and are sensitive to various abiotic stresses and ABA treatment in rice.

\section{Other Abiotic Stress Responsive cis-Acting Elements in Rice}

$\mathrm{ABRE}$ is also responsive to other categories of abiotic stresses like drought and salinity. In case of rice $\operatorname{rab} 16 A-D$, the ABRE (RTACGTGGCR) was reported to be desiccation stress responsive (Mundy et al., 1990). Among the other prominent groups of abiotic stress responsive cis-elements which can activates gene expression in the absence of ABA are termed as DRE/CRT, originally identified in Arabidopsis RD29A/COR78/LTI78 promoter (Table-2). This CRE is responsive to high salinity, drought and low temperature (YamaguchiShinozaki and Shinozaki, 2006). The regulatory activity of DRE/CRT elements was also found to be conserved in rice. The OsDREB1A protein binds to GCCGAC core of the DRE/CRT element more preferentially than to the ACCGAC, whereas the DREB1A protein binds to both GCCGAC and ACCGAC efficiently (Dubouzet et al., 2003). The cold responsive hexamer motif sequence (ACGTCA) was reported to be present in LIP19 promoters of rice (Table-2) to which $O B F 1$ that encodes a bZIP protein (OsOBF1) interacts and regulates low-temperature responsive gene expression (Shimizu et al., 2005). CORE (coordinate regulatory element for antioxidant defence) was found to play an important role in response to oxidative stress in rice (Tsukamoto et al., 2005). The core sequence of the OsIRO2-binding sequence (CACGTGG) occurred more frequently in the upstream regions of Fe deficiency-inducible genes than in the corresponding regions of non-inducible genes. OsIRO2 is a putative basic helix-loop-helix (bHLH) transcription factor encoding gene, 
which was strongly expressed in both roots and shoots during Fe deficiency stress (Ogo et al., 2006).

\section{Conclusions}

Abiotic stress responsive cis-elements are important regulators of transcriptional gene regulation and therefore important hallmark of abiotic stress inducible promoters. Well characterised abiotic stress inducible promoter elements have tremendous potential for engineering crop plants with improved tolerance to adverse environmental factors.

\section{References}

Abe, H., Yamaguchi-Shinozaki, K., Urao, T., Iwasaki, T., Hosokawa, D., and Shinozaki, K. (1997) Role of Arabidopsis MYC and MYB homologs in drought- and abscisic acid-regulated gene expression. Plant Cell, 9(10), 1859-1868.

Aoyama, T. (1999) Glucocorticoid induced gene expression in plants. Inducible gene expression in plants (Reynolds P. H. S. ed) Printed in the UK at the University Press, Cambridge: CAB International

Bhatnagar-Mathur, P., Vadez, V., and Sharma, K.K. (2008) Transgenic approaches for abiotic stress tolerance in plants: retrospect and prospects. Plant Cell Rep, 27(3), 411-424.

Bohnert, H.J., Nelson, D.E., and Jensen, R.G. (1995) Adaptations to environmental stresses. Plant Cell, 7(7), 1099-1111.

Boyer, J.S. (1982) Plant productivity and environment. Science, 218(4571), 443-448.

Burgess-Beusse, B., Farrell, C., Gaszner, M., Litt, M., Mutskov, V., Recillas-Targa, F., Simpson, M., West, A., and Felsenfeld, G. (2002) The insulation of genes from external enhancers and silencing chromatin. Proc Natl Acad Sci U S A, 99 Suppl 4, 16433-16437 Busk, P.K., and Pages, M. (1998) Regulation of abscisic acid-induced transcription. Plant Mol Biol, 37(3), 425-435.

Bustos, MM, Begum, D, Kalkan, FA et al (1991) Positive and negative cis-acting DNA domains are required for spatial and temporal regulation of gene expression by a seed storage protein promoter. EMBO J 10: 1469-1479.

Chinnusamy, V, Ohta, M, Kanrar, S et al (2003) ICE1: a regulator of cold-induced transcriptome and freezing tolerance in Arabidopsis. Genes Dev 17: 1043-1054.

Chinnusamy, V, Schumaker, K, Zhu, JK (2004) Molecular genetic perspectives on cross-talk and specificity in abiotic stress signaling in plants. $J$ Exp Bot 55: 225-236.

Dubouzet, J.G., Sakuma, Y., Ito, Y., Kasuga, M., Dubouzet, E.G., Miura, S., Seki, M., Shinozaki, K., and Yamaguchi-Shinozaki, K. (2003) OsDREB genes in rice, Oryza sativa L., encode transcription activators that function in drought-, high-salt- and cold-responsive gene expression. Plant J, 33(4), 751-763.

Guiltinan, M.J., Marcotte, W.R., Jr., and Quatrano, R.S. (1990) A plant leucine zipper protein that recognizes an abscisic acid response element. Science, 250(4978), 267-271.

Hakimi, MA, Privat, I, Valay, JG et al (2000) Evolutionary conservation of C-terminal domains of primary sigma (70)-type transcription factors between plants and bacteria. $J$ Biol Chem 275: 9215-9221. 
Hartmann, U, Sagasser, M, Mehrtens, F et al (2005) Differential combinatorial interactions of cis-acting elements recognized by R2R3-MYB, BZIP, and BHLH factors control lightresponsive and tissue-specific activation of phenylpropanoid biosynthesis genes. Plant Mol Biol 57: 155-171.

Hattori, T, Totsuka, M, Hobo, T et al (2002) Experimentally determined sequence requirement of ACGT-containing abscisic acid response element. Plant Cell Physiol 43: 136-140.

Hauffe, K.D., Lee, S.P., Subramaniam, R., and Douglas, C.J. (1993) Combinatorial interactions between positive and negative cis-acting elements control spatial patterns of 4CL1 expression in transgenic tobacco. Plant J, 4(2), 235-253.

Hirt, H, Kogl, M, Murbacher, T et al (1990) Evolutionary conservation of transcriptional machinery between yeast and plants as shown by the efficient expression from the CaMV $35 \mathrm{~S}$ promoter and 35S terminator. Curr Genet 17: 473-479.

Hobo, T., Asada, M., Kowyama, Y., and Hattori, T. (1999) ACGT-containing abscisic acid response element (ABRE) and coupling element 3 (CE3) are functionally equivalent. Plant $J$, 19(6), 679-689.

Hwang, Y.S., Karrer, E.E., Thomas, B.R., Chen, L., and Rodriguez, R.L. (1998) Three ciselements required for rice alpha-amylase Amy3D expression during sugar starvation. Plant Mol Biol, 36(3), 331-341.

Joshee, N., Kisaka, H., and Kitagawa, Y. (1998) Isolation and characterization of a water stress-specific genomic gene, pwsi 18, from rice. Plant Cell Physiol, 39(1), 64-72.

Latchman, D.S. (1997) Transcription factors: an overview. Int J Biochem Cell Biol, 29(12), 1305-1312.

Lenka, S.K., Lohia, B., Kumar, A., Chinnusamy, V., and Bansal, K.C. (2009) Genome-wide targeted prediction of ABA responsive genes in rice based on over-represented cis-motif in coexpressed genes. Plant Mol Biol, 69(3), 261-271.

Li, S., Assmann, S.M., and Albert, R. (2006) Predicting essential components of signal transduction networks: a dynamic model of guard cell abscisic acid signaling. PLoS Biol, 4(10), e312.

Mittler, R. (2006) Abiotic stress, the field environment and stress combination. Trends Plant

Sci, 11(1), 15-19.

Moons, A., De Keyser, A., and Van Montagu, M. (1997) A group 3 LEA cDNA of rice, responsive to abscisic acid, but not to jasmonic acid, shows variety-specific differences in salt stress response. Gene, 191(2), 197-204.

Morris, P.C., Kumar, A., Bowles, D.J., and Cuming, A.C. (1990) Osmotic stress and abscisic acid induce expression of the wheat Em genes. Eur J Biochem, 190(3), 625-630.

Mundy, J., Yamaguchi-Shinozaki, K., and Chua, N.H. (1990) Nuclear proteins bind conserved elements in the abscisic acid-responsive promoter of a rice rab gene. Proc Natl Acad Sci U S A, 87(4), 1406-1410.

Mundy, J., Yamaguchi-Shinozaki, K., and Chua, N.H. (1990) Nuclear proteins bind conserved elements in the abscisic acid-responsive promoter of a rice rab gene. Proc Natl Acad Sci US A, 87(4), 1406-1410.

Ogo, Y., Itai, R.N., Nakanishi, H., Inoue, H., Kobayashi, T., Suzuki, M., Takahashi, M., Mori, S., and Nishizawa, N.K. (2006) Isolation and characterization of IRO2, a novel iron-regulated bHLH transcription factor in graminaceous plants. J Exp Bot, 57(11), 2867-2878.

Ono, A., Izawa, T., Chua, N.H., and Shimamoto, K. (1996) The rab16B promoter of rice contains two distinct abscisic acid-responsive elements. Plant Physiol, 112(2), 483-491.

Qureshi, M.I., Qadir, S., and Zolla, L. (2007) Proteomics-based dissection of stress-responsive pathways in plants. Journal of Plant Physiology, 164(10), 1239-1260.

Reynolds, P.H.S. (1999) Inducible control of gene expression: an overview. Inducible gene expression in plants (Reynolds P. H. S. ed) Printed in the UK at the University Press, 
Cambridge: CAB International, 1-9.

Ross, C., and Shen, Q.J. (2006) Computational prediction and experimental verification of HVA1-like abscisic acid responsive promoters in rice (Oryza sativa). Plant Mol Biol, 62(1-2), 233-246.

Seo, H.S., Kim, H.Y., Jeong, J.Y., Lee, S.Y., Cho, M.J., and Bahk, J.D. (1995) Molecular cloning and characterization of RGA1 encoding a G protein alpha subunit from rice (Oryza sativa L. IR-36). Plant Mol Biol, 27(6), 1119-1131.

Shen, Q, Ho, TH (1995) Functional dissection of an abscisic acid (ABA)-inducible gene reveals two independent ABA-responsive complexes each containing a G-box and a novel cisacting element. Plant Cell 7: 295-307.

Shen, Q., Zhang, P., and Ho, T.H. (1996) Modular nature of abscisic acid (ABA) response complexes: composite promoter units that are necessary and sufficient for ABA induction of gene expression in barley. Plant Cell, 8(7), 1107-1119.

Shimizu, H., Sato, K., Berberich, T., Miyazaki, A., Ozaki, R., Imai, R., and Kusano, T. (2005) LIP19, a basic region leucine zipper protein, is a Fos-like molecular switch in the cold signaling of rice plants. Plant Cell Physiol, 46(10), 1623-1634.

Singh, KB (1998) Transcriptional regulation in plants: the importance of combinatorial control. Plant Physiol,118: 1111-1120.

Skriver, K., Olsen, F.L., Rogers, J.C., and Mundy, J. (1991) cis-acting DNA elements responsive to gibberellin and its antagonist abscisic acid. Proc Natl Acad Sci U S A, 88(16), 7266-7270.

Tran, LS, Nakashima, K, Shinozaki, K, et al (2007) Plant gene networks in osmotic stress response: from genes to regulatory networks. Methods Enzymol 428: 109-128.

Tsukamoto, S., Morita, S., Hirano, E., Yokoi, H., Masumura, T., and Tanaka, K. (2005) A novel cis-element that is responsive to oxidative stress regulates three antioxidant defense genes in rice. Plant Physiol, 137(1), 317-327.

Venter, M. (2007) Synthetic promoters: genetic control through cis engineering. Trends Plant Sci, 12(3), 118-124.

Viswanathan, C., and Zhu, J.K. (2002) Molecular genetic analysis of cold-regulated gene transcription. Philos Trans R Soc Lond B Biol Sci, 357(1423), 877-886.

Yamaguchi-Shinozaki, K, Shinozaki, K (2005) Organization of cis-acting regulatory elements in osmotic- and cold-stress-responsive promoters. Trends Plant Sci 10: 88-94.

Yamaguchi-Shinozaki, K., and Shinozaki, K. (2006) Transcriptional regulatory networks in cellular responses and tolerance to dehydration and cold stresses. Annu Rev Plant Biol, 57, 781-803.

Yamaguchi-Shinozaki, K., Mundy, J., and Chua, N.H. (1990) Four tightly linked $r a b$ genes are differentially expressed in rice. Plant Mol Biol, 14(1), 29-39.

Zhang, Z.L., Xie, Z., Zou, X., Casaretto, J., Ho, T.H., and Shen, Q.J. (2004) A rice WRKY gene encodes a transcriptional repressor of the gibberellin signaling pathway in aleurone cells. Plant Physiol, 134(4), 1500-1513.

Zhou, J, Wang, X, Jiao, Y et al (2007) Global genome expression analysis of rice in response to drought and high-salinity stresses in shoot, flag leaf, and panicle. Plant Mol Biol 63: 591608. 
Table-1 ABA responsive cis-acting elements containing ACGT/non ACTG sequence

\begin{tabular}{|c|c|c|}
\hline $\begin{array}{l}\text { Sequence of the } \mathrm{ABA} \\
\text { responsive } \text { cis-element }\end{array}$ & Promoter(s) & Reference \\
\hline ACGTACGTGTCG & empl & Morris et al.1990 \\
\hline RTACGTGGCR & rabl6 & Mundy et al.1990 \\
\hline ACATACGTGGCGG & $r a b 16 c$ & $\begin{array}{l}\text { Yamaguchi-Shinozaki et al. } \\
\text { (1990) }\end{array}$ \\
\hline ACGTACGTGGCGC & rabl6d & $\begin{array}{l}\text { Yamaguchi-Shinozaki et al. } \\
\text { (1990) }\end{array}$ \\
\hline CAGT $\underline{A C G T G G C A G}$ & $r a b 16 b$ & $\begin{array}{l}\text { Yamaguchi-Shinozaki et al. } \\
\text { (1990) }\end{array}$ \\
\hline CCGTACGTGGCGC & rabl6a & $\begin{array}{l}\text { Yamaguchi-Shinozaki et al. } \\
\text { (1990) }\end{array}$ \\
\hline GTACGTGGCGC & $\begin{array}{l}\text { rabl6 and alpha- } \\
\text { amylase genes }\end{array}$ & Skriver et al. (1991) \\
\hline $\begin{array}{l}\text { ACGTG-containing } \\
\text { sequences }\end{array}$ & Osem & Hattori et al.(1995) \\
\hline CCACGTGG & rgal & Seo et al. (1995) \\
\hline AGTACGTGGC & $r a b 16 b$ & Ono et al. (1996) \\
\hline GCCGCGTGGC & $r a b 16 b$ & Ono et al. (1996) \\
\hline CACATG & $r d 22$ & Abe et al. (1997) \\
\hline TGGTTAG & $r d 22$ & Abe et al. (1997) \\
\hline TGCTACGTGTCCC & lea & Moons et al. (1997) \\
\hline TTCTACGTGGCCA & lea & Moons et al. (1997) \\
\hline ACGTSSSC & $r a b 21$ & Busk and Pages (1998) \\
\hline CTACGTGGCCA & amy3d & Hwang et al. (1998) \\
\hline CCCTACGTGGCCG & wsil8 & Joshee et al.(1998) \\
\hline AACGCGTGTC & $V p 1$ & Hobo et al. (1999) \\
\hline ACGTGKC & Osem & Hattori et al. (2002) \\
\hline CTCCACGTGGCAC & similar to $H v a 22$ & Zhang et al. (2004) \\
\hline TACCACGTGGCAT & $t b 2 / d p 1 H v a 22$ & Zhang et al. (2004) \\
\hline$\underline{\text { ACGTGGC }}$ & $\begin{array}{l}\text { Hval rice } \\
\text { orthologue }\end{array}$ & Ross and Shen (2006) \\
\hline CGMCACGTGB & genome wide & Lenka et al. (2009) \\
\hline
\end{tabular}


Table-2 Abiotic stress responsive elements other than ABRE

\begin{tabular}{|l|l|l|l|}
\hline $\begin{array}{l}\text { Sequence of } \\
\text { different abiotic } \\
\text { stress responsive } \\
\text { cis-elements }\end{array}$ & $\begin{array}{l}\text { Motif/Promoter } \\
\text { name }\end{array}$ & $\begin{array}{l}\text { Abiotic Stress } \\
\text { Condition }\end{array}$ & Reference \\
\hline RTACGTGGCR & rabl6A-D & Desiccation & Mundy et al. (1990) \\
\hline GCCGAC & DRE/CRT & $\begin{array}{l}\text { Drought-, high salt- } \\
\text { and cold }\end{array}$ & Dubouzet et al. (2003) \\
\hline ACGTCA & LIP19 & Cold & Shimizu et al. (2005) \\
\hline $\begin{array}{l}\text { AAKAATWYRT } \\
\text { AWATAAAAM } \\
\text { TTTTATWTA }\end{array}$ & CORE & Oxidative stress & $\begin{array}{l}\text { Tsukamoto et al. } \\
(2005)\end{array}$ \\
\hline CACGTGG & $\begin{array}{l}\text { OsIRO2-binding } \\
\text { sequence }\end{array}$ & Fe deficiency stress & Ogo et al. (2006) \\
\hline
\end{tabular}

